\title{
The Relationship Between Sleep and Physical Activity Level in Barth Syndrome: A Cross-Sectional Analysis
}

\author{
Neil A. Judd*, Hannah S. Calhoun, Virginia Chu, Stacey Reynolds
}

Virginia Commonwealth University

\section{Article Info}

\section{Article Notes}

Received: July 18, 2020

Accepted: September 16, 2020

\section{${ }^{*}$ Correspondence:}

Dr. Neil A. Judd, Virginia Commonwealth University, 907 Floyd Ave, Richmond, VA 23284, United States ; Email: juddna@mymail.vcu.edu

C 2020 Judd NA. This article is distributed under the terms of the Creative Commons Attribution 4.0 International License.

\section{Keywords:}

Barth syndrome

sleep latency

sleep efficiency

nighttime awakenings

energy expenditure

actigraphy

\begin{abstract}
Barth syndrome (BTHS) is a rare genetic disorder commonly characterized by cardiomyopathy, muscle weakness, abnormal fatigability, and exercise intolerance. Sleep problems have been identified informally within the BTHS community though it has never been systematically studied. This study aimed to (1) objectively quantify sleep in males with BTHS, (2) examine the relationship between sleep and physical activity levels, and (3) examine agerelated trends in data. Twenty participants ( 7 adults and 13 children) with BTHS completed the study. Each participant wore an Actigraph GT9X activity tracker and completed a sleep diary for 14 consecutive days. Energy expenditure was calculated at various time periods: early morning, late morning, early afternoon, late afternoon, early evening, and late evening. Generalized Linear Models support statistically significant interactions between physical activity levels throughout the day and sleep parameters (sleep latency, efficiency, and nighttime awakenings). Trend analysis showed children had a longer sleep latency, higher sleep efficiency, and more nighttime awakenings than adults and were more active overall. Some of the findings in our study contradict current literature pointing to a relationship between sleep and physical activity levels in the typical population. This suggests other factors may impact decreased sleep in BTHS and warrants future studies into the causes of decreased sleep and the relationship to physical activity in this population.
\end{abstract}

\section{Introduction}

\section{Background}

Barth syndrome (BTHS) is an X-linked genetic disorder affecting approximately 150-200 living boys and men worldwide; although rare, evidence is accumulating that the disorder is underdiagnosed. ${ }^{1}$ The primary defect in BTHS is a disordered remodeling of cardiolipin, a principal phospholipid in the inner mitochondrial membrane. In BTHS, defects in cardiolipin remodeling have been shown to increase the number of abnormal mitochondria in cells and impact electron transport chain formation and function. ${ }^{2}$ It is currently understood that BTHS is caused by a mutation or deletion of the tafazzin (TAZ) gene, located at Xq28, though several different mutations have been identified leading to variable clinical presentations. ${ }^{3}$ The clinical phenotype of BTHS is most commonly characterized by cardiomyopathy, left ventricular non-compaction, skeletal myopathy (predominantly proximal muscle weakness), neutropenia, and 3-methylglutaconic aciduria. Additional features include delayed motor milestones, abnormal fatigability, and exercise intolerance. ${ }^{1}$

Sleep problems have been identified informally within the BTHS community (Shelley Bowen, personal communication), though sleep 
Table 1. Participant Characteristics

\begin{tabular}{|c|c|c|c|c|c|c|}
\hline \multirow{4}{*}{ Participants } & \multicolumn{2}{|c|}{ Adults } & \multicolumn{2}{|c|}{ Children } & \multirow{2}{*}{\multicolumn{2}{|c|}{$\begin{array}{c}\text { Total } \\
\mathbf{n}\end{array}$}} \\
\hline & \multirow{3}{*}{$\begin{array}{c}\text { n } \\
7 \\
\text { Mean (SD) }\end{array}$} & \multirow{3}{*}{\begin{tabular}{c|}
$\%$ \\
$35 \%$ \\
Range \\
\end{tabular}} & \multirow{3}{*}{$\begin{array}{c}\mathbf{n} \\
13 \\
\text { Mean (SD) }\end{array}$} & \multirow{3}{*}{\begin{tabular}{|c|}
$\%$ \\
$65 \%$ \\
Range \\
\end{tabular}} & & \\
\hline & & & & & \multicolumn{2}{|c|}{20} \\
\hline & & & & & Mean (SD) & Range \\
\hline Age (years) & $28.64(5.41)$ & $20.0-37.1$ & $12.42(3.34)$ & 7.7 - 17.7 & $18.10(9.02)$ & $7.7-37.1$ \\
\hline Weight (kg) & $63.26(17.47)$ & $36.6-87.6$ & $33.24(12.13)$ & $17.9-52.7$ & $43.74(20.66)$ & $17.9-87.6$ \\
\hline
\end{tabular}

has never been systematically studied in this population. Several factors put males with BTHS at risk for sleep problems. First, many individuals with genetically caused disorders have sleep problems regardless of the mutation. ${ }^{4}$ Second, sleep problems are more prevalent in individuals with genetic mutations and learning differences. Learning differences, particularly in math have been noted in the BTHS population which puts them in this higher risk group..$^{5}$ Finally, daytime physical activity and refraining from naps has been shown to be a part of a good sleep hygiene routine; however, males with BTHS are often limited in the amount of daytime physical activity they can tolerate and are prone to daytime napping due to high fatigue levels. ${ }^{1,6}$

Our rationale for this study was that individuals with BTHS are predisposed to have sleep problems due to their genetic condition and associated limitations in physical activity. Our study was the first to examine sleep in BTHS and provides a basis for future efforts to improve the quality of life for this population.

\section{Objectives}

The aims of the study were to (1) objectively quantify sleep and sleep problems in a sample of males with BTHS, (2) examine the relationship between sleep and physical activity levels in individuals with BTHS, and (3) examine age-related trends in sleep and physical activity data. We hypothesized that individuals with BTHS would have evidence of sleep difficulties (i.e., increased sleep onset latency, decreased sleep efficiency, and high number of nighttime awakenings) and low levels of physical activity throughout the day. Additionally, we hypothesized that higher physical activity levels during the afternoon would correlate with reduced sleep disturbances. ${ }^{7}$

\section{Methods}

\section{Study Design and Overview}

This study used a non-experimental, cross-sectional, study design to objectively measure sleep and physical activity patterns across a period of two weeks in males with BTHS. This project was part of a larger research study comprehensively examining sleep problems in this population.

\section{Participants and Recruitment}

A convenience sample of 21 males diagnosed with
Barth aged 7.7 - $37.1(M=18.10 ; S D=9.02)$ were enrolled in the study, however one participant was excluded due to incomplete data. Table 1 includes only those participants for which data was included $(n=20)$. Individuals were eligible for study inclusion if they: (i) had a medically verified diagnosis of Barth syndrome and (ii) were aged 7 and above. Exclusion criteria were as follows: (i) individual or caregiver unable to consent and complete forms in English, (ii) children who were wards of the state, and (iii) individuals with comorbid physical disabilities (e.g., cerebral palsy).

Initial study recruitment occurred during the 2018 International Barth Syndrome Foundation Scientific and Medical Family Conference in Clearwater Beach, FL. An informational session was held to provide individuals and families an opportunity to learn about the study. Part of this informational session included a question/answer portion between potential participants and researchers. Once families and individuals had all of their questions answered, consent forms were signed on site; children between 7-18 years of age provided written assent. All study procedures were approved by the sponsoring university's institutional review board.

\section{Procedures}

Conference coordinators screened all interested families for inclusion criteria. Training sessions were scheduled with the primary investigator during the 2018 conference; during these sessions all inclusion and exclusion criteria were verified verbally with individuals with BTHS and/or their families. Since this study used actigraphy and a sleep diary to track sleep, training was provided at the conference for proper care and charging of the activity tracker (Actigraph GT9X Link) and how to accurately complete the provided sleep diary. At the end of the conference, seven individuals took home an Actigraph GT9X Link, charging cable, charging dock, instruction booklet, a sleep diary, contact information for study coordinators, a return envelope as well as a prepaid and pre-addressed Fed-Ex return label. The remaining 13 individuals who consented to participate, were mailed study materials as they became available over the next 6 months.

Each participant was instructed to wear the Actigraph GT9X Link on his wrist and complete the sleep diary for 14 days and mail all study materials back to investigators once completed. The serial number from each Actigraph 
was recorded and linked to each participant on a separate code document in order to track each Actigraph GT9X Link activity tracker.

\section{Materials and Measures}

Actigraph GT9X Link. The activity tracker, ActiGraph GT9X Link (henceforth referred to only as GT9X), was used to collect sleep and physical activity data for the duration of this study. The GT9X is a wrist-worn activity tracker that utilizes a 3-axis accelerometer to collect sleep and physical activity data. The GT9X was initialized using ActiLife software (Version 6, ActiGraph, LLC., Pensacola FL) to collect data at a sample rate of $30 \mathrm{~Hz}$, and data was uploaded to ActiLife at 60 -second epochs. Sleep and wake times were automatically identified using the Sadeh algorithm, which has been validated for pediatric and adult populations. $^{8}$

The following sleep variables were measured using the GT9X: sleep onset latency, sleep efficiency, and number of nighttime awakenings. Sleep onset latency was defined as the length of time between getting in bed and falling asleep and was recorded in minutes. Nighttime awakenings were numerically recorded as any disruption to a sleep episode lasting at least 60 seconds. Sleep efficiency was defined as the number of sleep minutes divided by the total number of minutes the subject was in bed in percentage format.

Sleep Diary. A sleep diary which documented the estimated time the participant got into bed, fell asleep, awoke the next morning, whether or not a nap was taken that day, as well as any additional comments was provided to each participant to be completed daily. Sleep diaries were sent with detailed instructions for each recording parameter as well as instruction to record time per watch (set to EST). Instructions provided with sleep diaries included the following:

- What time did you (or your child) wake up in the morning? This will be the time that you fully woke up with no further attempts at going back to sleep.

- What time did you (or your child) get into bed? Write the time you got into bed. This may not be the time that you began "trying to fall asleep".

- What time did you (or your child) try to go to sleep? This is usually when you turn the lights out and any devices off.

- Did you (or your child) nap today? If yes, how long? If you took a nap, please write down approximately what time you started napping and what time you woke up.

- Comments: If you have anything else that you would like to say that is relevant to your sleep, please feel free to write it here.
Diaries were completed by each participant in a written format; if child participants were unable to reliably record diary information, parents were instructed to complete the sleep diary for their child. Additionally, sleep diaries were used to adjust and validate the in-bed and out-bed times in the Actilife software (ActiGraph LLC, Pensacola, Florida) to ensure that sleep periods and sleep variables were accurate.

Energy Expenditure. The GT9X gathered physical activity counts per minute (CPM) and vector magnitude (VM) for each epoch (60 second time-interval) during the entire 14 days of data collection. CPM is calculated from the summation axis 1 , axis 2 , and axis 3 whereas the formula for $\mathrm{VM}$ is the square root of all 3 axes squared and refers to the magnitude of the triaxial vector (ActiLife 6 User's Manual). We utilized the algorithm developed by Romero-Ugalde and colleagues to determine average energy expenditure (kcals/minute) using weight $(\mathrm{kg}$ ) and counts per minute or vector magnitude. ${ }^{9}$ Average energy expenditure was calculated for the following time frames: early morning (EM) from 6am to 8:59am, late morning (LM) from 9am to 11:59am, early afternoon (EA) from $12 \mathrm{pm}$ to $2: 59 \mathrm{pm}$, late afternoon (LA) from 3pm to 5:59pm, early evening (EEVE) from $6 \mathrm{pm}$ to $8: 59 \mathrm{pm}$, and late evening (LEVE) from 9pm to $11: 59 \mathrm{pm}$. All times were recorded in Eastern Standard Time (EST). The above energy expenditure analysis was conducted using methodology described by Calhoun et al. ${ }^{10}$

\section{Data Analysis}

To test our first objective, we used descriptive statistics to determine average sleep onset latency, sleep efficiency, and number of nighttime awakenings for each participant. To test our second objective, we used Microsoft Excel and IBM SPSS Statistics Version 26 to analyze sleep and energy expenditure data. We ran generalized linear models (GLMs) in SPSS to determine the significance and relationship of participants' energy at each time period against three sleep variables (sleep latency, sleep efficiency, and nighttime awakenings). GLMs were conducted for all participants enrolled in study, adult participants, and child participants to ascertain trends across age. Each GLM was analyzed using the Test of Model Effects and Parameter Estimates output tables with a $p$ value of .05. We used MANOVAs and descriptive statistics to test our third objective of examining age related trends in sleep and physical activity data. For all analyses, data was excluded if less than $80 \%$ was available per variable; based on this criterion, day 14 was excluded from further analysis for all subjects.

Participant daytime naps reported on sleep diaries were not included in further statistical analysis because no naps were recorded on the GT9X actigraphs. It is important to note that of the 20 total participants, only five $(1$ child, 4 adults) self-reported taking naps throughout the entirety 
of the study; however, none of these reported naps were verifiable through the GT9X software and were therefore excluded from further analysis.

\section{Results}

\section{Total Sample}

Individuals diagnosed with Barth syndrome who participated in this study had an average sleep onset latency of 36.28 minutes (range 0-327 minutes), an average sleep efficiency of $73.29 \%$ (range $31.5-99.82 \%$ ), and an average number of nighttime awakenings of 24.79 (range 0-50 times). Highest energy expenditure during for the total sample was in late afternoon (3pm-5:59pm EST) with a mean of $652.59 \mathrm{kcals} / \mathrm{min}$ (range $0-2370.24 \mathrm{kcals} / \mathrm{min}$ ) and the lowest energy expenditure during early morning (6am-8:59am EST) with a mean of $204.93 \mathrm{kcals} / \mathrm{min}$ (range 0-1261.81 kcals/min). Average total energy expenditure per day was $2845.1 \mathrm{kcals} / \mathrm{min}$ (range 49.2-7386.99 kcals/ $\mathrm{min}$ ). Per GLM results there was a statistically significant interaction between nighttime awakenings and early morning ( $p=.01, B=-.007)$, late afternoon ( $p=.016, B=.004)$, and late evening ( $p=.002, B=-.007)$ energy expenditure. Results indicate that increased energy expenditure during early morning and late evening decreased the occurrence of nighttime awakenings; conversely increased energy expenditure during late afternoon increased the number of nighttime awakenings (See Tables 2 and 3 and Figure 1). There were no statistically significant relationships between energy expenditure and sleep latency or sleep efficiency.

Table 2. Sleep and Energy Expenditure Demographics

\begin{tabular}{|c|c|c|c|c|c|c|}
\hline & \multicolumn{2}{|c|}{ Adults } & \multicolumn{2}{|c|}{ Children } & \multicolumn{2}{|c|}{ Total } \\
\hline & Mean (SD) & Range & Mean (SD) & Range & Mean (SD) & Range \\
\hline \multicolumn{7}{|l|}{ Sleep } \\
\hline Latency (min) & $25.56(35.39)$ & $0-211$ & $41.07(44.62)$ & $0-327$ & $36.28(42.37)$ & $0-327$ \\
\hline Efficiency (\%) & $71.86(15.16)$ & $35.77-97.21$ & $74.01(11.07)$ & $31.5-99.82$ & $73.29(12.6)$ & $31.5-99.82$ \\
\hline Awakenings* & $21.91(9.37)$ & $4-48$ & $26.26(9.27)$ & $0-50$ & $24.79(9.51)$ & $0-50$ \\
\hline \multicolumn{7}{|c|}{ Energy Expenditure (kcals/min) } \\
\hline EM & $292.84(321.46)$ & $0-1261.81$ & $160.45(193.47)$ & $0-1068.55$ & $204.93(251.35)$ & $0-1261.81$ \\
\hline LM & $584.24(509.69)$ & $0-1638.86$ & $461.61(337.76)$ & $0-1891.15$ & $502.81(406.9)$ & $0-1891.15$ \\
\hline EA & $522.13(428.38)$ & $0-1468.43$ & $675.61(447.5)$ & $0-2313.24$ & $624.04(446.27)$ & $0-2313.24$ \\
\hline LA & $513.69(414.25)$ & $0-1873.99$ & $722.86(475.25)$ & $0-2370.24$ & $652.59(465.49)$ & $0-2370.24$ \\
\hline EEVE & $389.58(331.44)$ & $6.59-1598.83$ & 651.07 (464.97) & $0-2255.12$ & $563.52(441.82)$ & $0-2255.12$ \\
\hline LEVE & $242.9(267.75)$ & $0-1407.82$ & $325.16(285.6)$ & $0-1748.6$ & $297.52(281.9)$ & $0-1748.6$ \\
\hline Total Energy/Day & $2545.37(1844.21)$ & $49.2-6912.74$ & 2996.75 (1394.49) & $284.02-7386.99$ & $2845.1(1570.9)$ & $49.2-7386.99$ \\
\hline
\end{tabular}

*number of nighttime awakenings

Table 3. Generalized Linear Model Results for Sleep and Physical Activity Levels

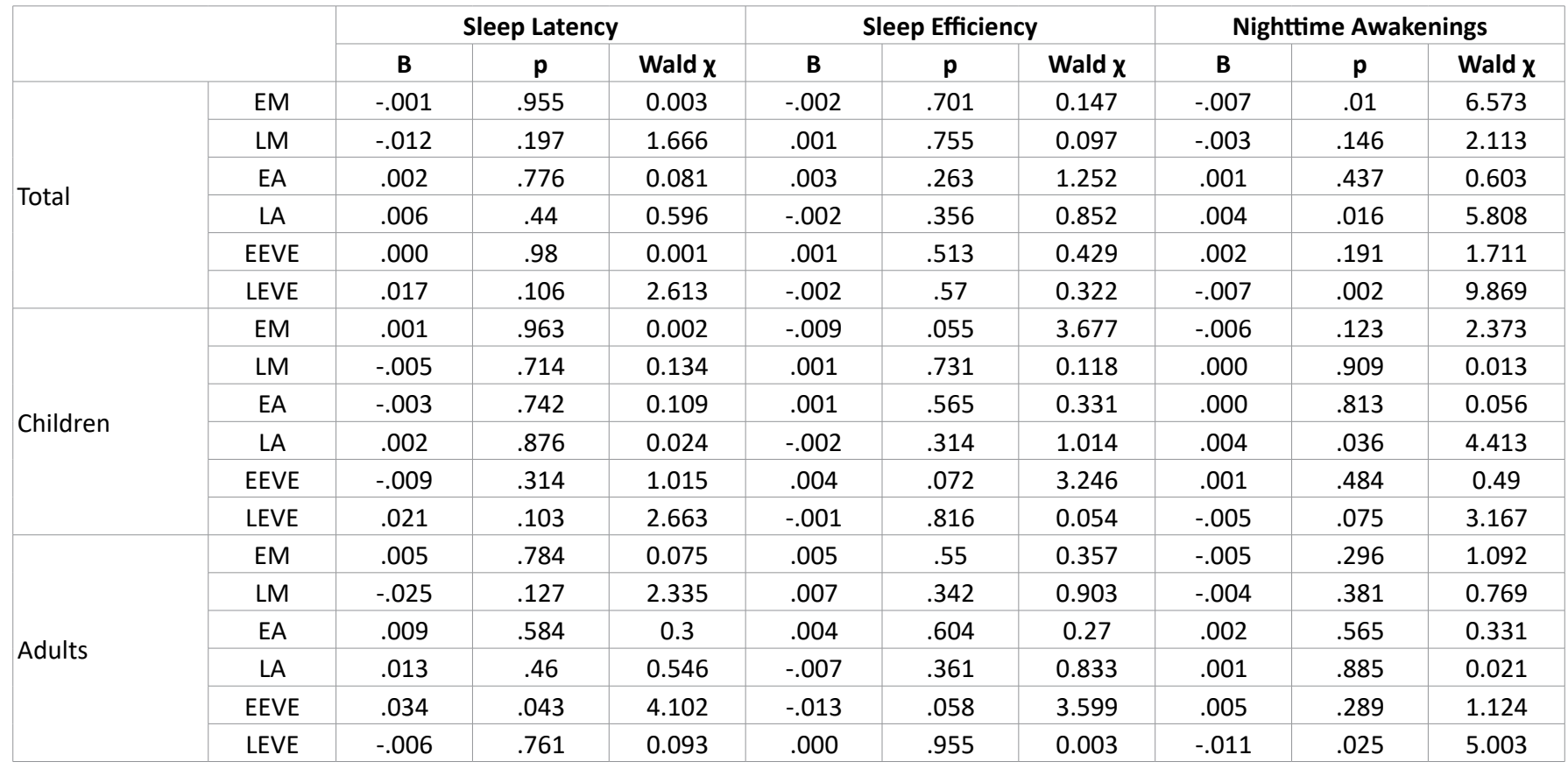




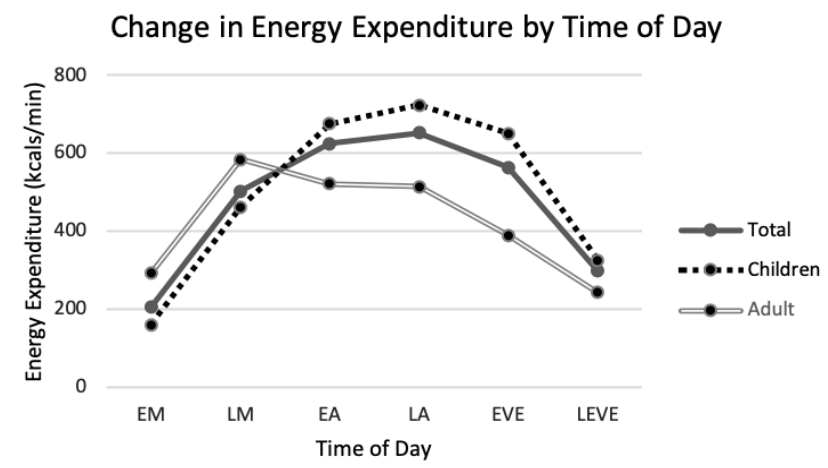

*EM (early morning), LM (late morning), EA (early afternoon), LA (late afternoon), EEVE (early evening), LEVE (late evening)

Figure 1. Average energy expenditure change by time of day for total participants, children, and adults

While important to look at the sample as a whole, we also examined differences between our child and adult samples. Results trended towards a higher sleep latency and increased occurrence of nighttime awakenings in children and a lower sleep efficiency in adults with Barth syndrome. Initial MANOVA results revealed a statistically significant difference in sleep variables based on age group $[F(3,16)=3.32, p=.047$; Wilk's $\Lambda=0.616$, partial $\eta 2=.38]$; though when examined through follow-up post-hoc testing, there was no actual statistically significant difference for sleep latency $[F(1,18)=1.815, p=.195$; partial $\eta 2=.092]$, sleep efficiency $[F(1,18)=.260, p=.616$; partial $\eta 2=.041]$, or nighttime awakenings $[F(1,18)=2.125, p=.162$; partial $\eta 2=.106$ ] between children and adults. Similarly, MANOVA results revealed there were no significant differences between the two groups (children and adults) for the timed energy expenditure variables included in analysis $[F(6,13)$ $=1.360, p=.301$; Wilk's $\Lambda=0.614$, partial $\eta 2=.386]$. While the difference was not statistically significant, children with BTHS expended slightly more total energy per day ( $M=2996.75 ; S D=1394.49)$ in comparison to adults in the study $(M=2545.37 ; S D=1844.21)$ and were most active in late afternoon, whereas adults were most active in late morning (Table 2). Overall, it appeared that adults were more active than children in early and late morning, but their physical activity levels steadily decreased throughout the remainder of the day. Children had a steady increase of physical activity from early morning to late afternoon, which then decreased in late evening (see Figure 1).

\section{Children}

Children included in the study sample had an average sleep onset latency of 41.07 minutes (range 0-327 minutes), an average sleep efficiency of $74.01 \%$ (range $31.5-99.82 \%$ ), and an average number of nighttime awakenings of 26.26 (range 0-50). Statistical analysis from the children in the study sample revealed the highest energy expenditure during late afternoon (3pm-5:59pm
EST) with a mean of $722.86 \mathrm{kcals} / \mathrm{min}$ (range 0-2370.24 $\mathrm{kcals} / \mathrm{min}$ ) and the lowest energy expenditure during early morning (6am-8:59am EST) with a mean of $160.45 \mathrm{kcals} /$ min (range 0-1068.44 kcals/min). Average total energy expenditure per day was 2996.75 (range 284.02-7386.99 kcals/min). Per GLM results, there was a statistically significant interaction between nighttime awakenings and late afternoon ( $p=.036, B=.004$ ) energy expenditure for child participants. Results indicate that increased energy expenditure during late afternoon increased the number of nighttime awakenings (See Tables 2 and 3 and Figure 1). There were no statistically significant relationships between energy expenditure and sleep latency or sleep efficiency, however energy expenditure during early morning approached statistical significance with sleep efficiency ( $p=.055, B=-.009$ ) which indicates increased amounts of physical activity in early morning may have a negative impact on sleep efficiency for children.

\section{Adults}

Adults included in the sample had an average sleep onset latency of 25.56 minutes (range 0-211 minutes), an average sleep efficiency of $71.86 \%$ (range 35.77 $97.21 \%$ ), and an average number of nighttime awakenings of 21.91 (range 4-48). Statistical analysis from the adult sample revealed the highest energy expenditure during late morning (9am-11:59am EST) with a mean of 584.24 $\mathrm{kcals} / \mathrm{min}$ (range 0-1638.86 kcals/min) and the lowest energy expenditure during late evening (9pm-11:59pm EST) with a mean of $242.9 \mathrm{kcals} / \mathrm{min}$ (range 0-1407.82 $\mathrm{kcals} / \mathrm{min}$ ). Average total energy expenditure per day was 2545.37 (range 49.2-6912.74 kcals/min). Per GLM results, there was a statistically significant interaction between nighttime awakenings and late evening ( $p=.025, B=-.011$ ) as well as early evening and sleep onset latency ( $p=.043$, $B=.034$ ) for adult participants. These results indicate that increased energy expenditure during late evening showed a decreased occurrence of nighttime awakenings and the more energy expended during early evening indicated an increased sleep onset latency (See Tables 2 and 3 and Figure 1) There were no significant relationships between energy expenditure and sleep efficiency, however energy expenditure during early evening approached a statistically significant interaction with sleep efficiency ( $p=.058, B$ =-.013; see Tables 2 and 3).

\section{Discussion}

Diagnostic indicators of insomnia include sleep efficiency below $85 \%$, sleep onset latency greater than 30 minutes, frequent nighttime awakenings ( $>18.5)$, and excessive daytime sleepiness. ${ }^{11,12,13,14}$ Sleep efficiency was found to be deficient in both adults (mean $=71.86 \%$ ) and children (mean $74.01 \%$ ) in this study. The time to fall asleep varied greatly amongst the adults in the study, 
with a range of 0-211 minutes across all days collected; overall, the sleep onset latency for adults in the study fell within the acceptable range with a mean of 25 minutes. ${ }^{12}$ Children, however, demonstrated a sleep onset latency of 41 minutes, with an even greater range of 0-327 minutes. Norms for nighttime awakenings are not well established, however, research by Bulckaert et al. suggest an average occurrence of nighttime awakenings in physically and mentally healthy men and women (with a mean age of 23 years) at 18.5 times per night. ${ }^{12}$ Adults in our sample averaged 21 nighttime awakenings, while children averaged 26 nighttime awakenings on average. Collectively, this data supports our hypothesis that individuals with BTHS exhibit sleep difficulties as evidenced by decreased sleep efficiency, increased sleep latency, and an increased incidence of nighttime awakenings. While sleep latency and frequency of nighttime awakenings appear to improve with age, our results suggest that sleep problems in BTHS that emerge in childhood may continue into adulthood without intervention.

The second aim of this study was to examine the relationship between sleep and physical activity levels in individuals with BTHS. Previous research and clinical guidelines suggest that increased levels of physical activity in the morning or afternoon (9am-6pm) increases sleep quality and quantity; late afternoon has even been suggested as an optimal time for higher levels of physical activity. ${ }^{7,15,16}$ Our hypothesis that higher physical activity levels during the afternoon would correlate with reduced sleep disturbances in BTHS was not supported. In fact, increased energy expenditure in the late afternoon correlated with increased frequency of nighttime awakenings in children with BTHS and in the total sample. Interestingly, increased energy expenditure during late evening improved sleep in adults (decreased nighttime awakenings). Late evening physical activity is typically not recommended to promote sleep due to increased body temperature created with physical activity. Decreased body temperature is needed for the brain to enter a sleep state and the body requires at least six hours after exercise to reach this optimal temperature zone. ${ }^{7}$ It is unclear whether the physical activity levels exerted by individuals with BTHS were high enough to significantly raise their body temperature during the study or whether their "cool down" time is faster than normal due to their genetic differences in metabolism. ${ }^{17}$ Possibly, adults with BTHS who exerted more late evening energy simply became fatigued enough to fall asleep; these are certainly important areas to consider for future research. It is currently unknown how constructs of physical and mental fatigue, exercise intolerance, and tiredness interact to impact sleep in individuals with genetic disorders. While proper timing of physical activity may be important to maximize sleep in individuals without metabolic or cardiac conditions, specific clinical guidelines for the BTHS population could look very different compared to guidelines for the general population.

\section{Conclusion}

Sleep is an important aspect of development and is critical for functioning throughout the day, for learning, and for overall health. Research indicates that poor sleep can lead to excessive daytime sleepiness and impaired motor and cognitive function, ultimately leading to an overall decrease in functional performance. ${ }^{19,20,21}$ This was the first study to systematically assess sleep in males diagnosed with BTHS. Our study suggests that both adults and children with BTHS meet most clinical criteria for insomnia, with deficits noted in their ability to fall asleep and stay asleep, leading to an overall deficit in sleep efficiency. This is important to consider within the BTHS population which already struggles with challenges related to motor function and daytime fatigue. Treatments to improve sleep have the potential to enhance not only sleep quality and quantity but affect daytime performance in educational and occupational tasks as well. Interventions will need to consider the unique features of BTHS, and acknowledge that common sleep hygiene recommendations, such as increasing afternoon physical activity, may not be effective for this population.

\section{Limitations}

A primary limitation of this study is unclear documentation of time on sleep diaries due to individuals participating in different time zones. Time zones were adjusted by the researchers, however there is room for human error in adjustments. Additional limitations include the inability to account for personal, temporal, and situational contexts. While the research indicates a relationship between sleep and physical activity levels in this sample, other factors may have influenced sleep, such as medication, sugar intake, sleep environment, sleep apnea, illness, etc. While our sample size was small $(N=20)$, our sample represented approximately $10 \%$ of those people currently living with BTHS suggesting high external validity. Additionally, actigraphs have potential to underestimate WASO as they tend to record "lying still, but awake" as "sleep"; this could indicate that sleep disturbances are even more prominent in this population than our data shows.

\section{Implications for Future Research}

Future research should further examine the relationship between physical activity levels and potential sleep difficulties across age groups to generate more comprehensive sleep and physical activity changes across thelifespan in individuals with BTHS. Wealso recommended that future research compare sleep and energy expenditure 
data between those diagnosed with BTHS and the typically developing population to determine if sleep or physical activity is different amongst populations and to corroborate our findings of overall decreased physical activity levels in individuals with BTHS. Comparison of BTHS with a normative sample is also recommended in order to generate activity recommendations for children and adults with BTHS; since our findings were the first of their kind to be published in BTHS, and have not yet been replicated, we were hesitant to make broad implications for practice at this current juncture. Future research should also focus on examining the determinants of decreased sleep in this population or other underlying conditions to account for the contradictory information between current research and data from our study.

\section{Acknowledgements}

We would like to acknowledge Shelly Bowen and Michaela Damin for their assistance with subject recruitment and Alex Calhoun for his assistance and guidance with data analysis. We would also like to thank the individuals with BTHS and their families who participated in this study. Special thanks to fellow occupational therapy students Emily Schuette, Heather Bouley, and Kaelah Pou, for their assistance in data collection.

\section{References}

1. Clarke, S. L. N., Bowron, A., Gonzalez I.L., Groves, S. J., Newbury-Ecob, R., Clayton, N... Steward, C. G. (2013) Barth syndrome. Orphanet Journal of Rare Disorders, 8, 1-17. doi: 10.1186/1750-1172-8-23

2. Gaspard, G. J. \& McMaster (2015). Cardiolipin metabolism and its causal role in the etiology of the inherited cardiomyopathy barth syndrome. Chemistry and Physics of Lipids, 193, 1-10. doi: 10.1016/j. chemphyslip.2015.09.005

3. Lu, Y. W., Galbraith, L., Herndon, J. D., Lu, Y. L., Pras-Rayes, M., ... \& Claypool, S. M. (2016). Defining functional classes of Barth syndrome mutation in humans. Human Molecular Genetics, 25 (9), 1754-1770. Retrieved from https://doi.org/10.1093/hmg/ddw046

4. Dorris, L., Scott, N., Zuberi, S., Gibson, N., \& Espie, C. (2008). Sleep problems in children with neurological disorders. Developmental Neurorehabilitation, 11(2), 95-114

5. Raches, D. \& Mazzocco, M. M. (2012). Emergence and nature of mathematical difficulties in young children with Barth syndrome. Journal of Developmental and Behavioral Pediatrics, 33(4), 328-335

6. National Sleep Foundation (2016). Sleep Hygiene. Retrieved from https://sleepfoundation.org/ask-the-expert/sleep-hygiene

7. National Sleep Foundation (2009). Diet, Exercise, and Sleep. Retrieved from https://www.sleepfoundation.org/articles/diet-exercise-and-sleep
8. Sadeh, A., Sharkey, K.M., \& Carskadon, M.A. (1994). Activity-based sleep-wake identification: an empirical test of methodological issues. Sleep, 17(3), 201-207

9. Romero-Ugalde, H., Garnotel, M., Doron, M., Jallon, P., Charpentier, G. \& Franc, S. (2017). An original piecewise model for computing energy expenditure from accelerometer and heart rate signals. Physiological Measurement, 38, 1599-1615. doi: 10.1088/1361-6579/aa7cdf

10. Calhoun, H. S., Judd, N. A., \& Chu, V. (in preparation). The relationship between sleep and activity levels in children: A case study methodology. [Unpublished manuscript]. Department of Occupational Therapy, Virginia Commonwealth University.

11. National Sleep Foundation (2020). Insomnia. Retrieved from https:// www.sleepfoundation.org/sleep-disorders/insomnia

12. Bulckaert, A., Exadaktylos, V., Bruyne G. D., Haex, B., Valck, E. D., Wuyts, J., Verbraecken, J., \& Berckmans, D. (2010). Heart rate-based nighttime awakening detection. European Journal of Applied Physiology, 109(2), 317-322. doi:10.1007/s00421-010-1359-0

13. Morin, C. M., Hauri, P. J., Espie, C. A., Spielman, A. J., Buysse, D. J., \& Bootzin, R. R. (1999). Nonpharmacologic treatment of chronic insomnia. SLEEP, 22(8), 1134-1156

14. Yu, J., Tseng, I., Tuan, R., Sheu, J., Liu, H., \& Hu, C. (2009). Low sleep efficiency in patients with cognitive impairment. Acta Neurologica Taiwanica, 18(2), 91-97

15. National Sleep Foundation (2018). How Exercise Impacts Sleep Quality. Retrieved from https://www.sleepfoundation.org/articles/ how-exercise-impacts-sleep-quality

16. Tatsumi, Y., Mohri, I., Shimizu, 2., Tachibana, N., Ohno, Y., \& Taniike, M. (2015). Daytime physical activity and sleep in pre-schoolers with developmental disorders. Journal of Paediatrics and Child Health, 51, 396-402. https://doi.ord/10.1111/jpc.12725

17. Cade, W. T., Laforest, R., Bohnert, K. L., Reeds, D. N., Bittel, A. J., de las Fuentes, L., Bashir, A., Woodard, P. K., Pacak, C. A., Byrne, B. J., Gropler, R. J., \& Peterson, L. R. (2019). Myocardial glucose and fatty acid metabolism are altered and associated with lower cardiac function in young adults with Barth syndrome. Journal of Nuclear Cardiology. doi:10.1007/s12350-019-01933-3

18. Zhu, Z., Chen, P., \& Zhuang, J. (2013). Predicting chinese children and youth's energy expenditure using actigraph accelerometers: A calibration and cross-validation study. Research Quarterly for Exercise and Sport, 84, S56-63. doi:10.1080/02701367.2013.850989

19. Annaz, D., Hill, C. M., Ashworth, A., Holley, S., \& Karmiloff-Smith, A. (2011). Characterisation of sleep problems in children with Williams syndrome. Research in Developmental Disabilities, 32(1), 164-169. doi:10.1016/j.ridd.2010.09.008

20. Curcio, G., Ferrara, M., \& Gennaro, L. D. (2006). Sleep loss, learning capacity and academic performance. Sleep Medicine Reviews, 10, 323337. doi: 10.1016/j.smrv.2005.11.001

21. Dewald, J. F., Meijer, A. M., Oort, F. J., Kerkhof, G. A., \& Bögels, S. M. (2010). The influence of sleep quality, sleep duration and sleepiness on school performance in children and adolescents: A metaanalytic review. Sleep Medicine Reviews, 14, 179-189. doi: 10.1016/j. smrv.2009.10.004 